\title{
SOME RESULTS ON HYPERCENTRAL UNITS IN INTEGRAL GROUP RINGS
}

\author{
Yuanlin Li and M.M. Parmenter ${ }^{1}$ \\ Department of Mathematics \\ Brock University \\ St. Catherines, Ontario, \\ Canada L2S 3A1 \\ Email: yli@brocku.ca \\ Department of Mathematics and Statistics \\ Memorial University of Newfoundland \\ St. John's, Newfoundland, \\ Canada A1C 5S7 \\ Email: mparmen@plato.ucs.mun.ca
}

\begin{abstract}
In this note we investigate the hypercentral units in integral group rings $\mathbb{Z} G$, where $G$ is not necessarily torsion. One of the main results obtained is the following (Theorem 3.5): if the set of torsion elements of $G$ is a subgroup $T$ of $G$ and if $Z_{2}(\mathcal{U})$ is not contained in $C_{\mathcal{U}}(T)$, then $T$ is either an Abelian group of exponent 4 or a $Q^{*}$ group. This extends our earlier result on torsion group rings.
\end{abstract}

\section{Introduction}

Let $\mathbb{Z} G$ denote the integral group ring of a group $G$ and $\mathcal{U}(\mathbb{Z} G)$ the group of units of such a group ring. For convenience, we will sometimes write $\mathcal{U}$ instead of $\mathcal{U}(\mathbb{Z} G)$. If $G$ is any group, let $Z_{n}(G)$ denote the n'th term of the ascending central series of $G$ and let $\tilde{Z}(G)=\cup Z_{n}(G)$, the hypercentre of $G$.

\footnotetext{
${ }^{1}$ This research was supported in part by grants from the Natural Sciences and Engineering Research Council.
} 
When $G$ is finite, Arora, Hales and Passi [1] showed that $\tilde{Z}(\mathcal{U})=Z_{2}(\mathcal{U})$ while Arora and Passi [2] showed that $\tilde{Z}(\mathcal{U}) \subseteq G \cdot Z(\mathcal{U})$ (and also completely determined $G \cap \tilde{Z}(\mathcal{U})$ ). These results were extended to torsion groups in [5] and [7].

Clearly, in general the equality, $\tilde{Z}(\mathcal{U})=Z_{2}(\mathcal{U})$, will no longer hold when $G$ is not necessarily torsion (consider torsion free nilpotent $G$ ). Our goal in this paper is to investigate the inequality $\tilde{Z}(\mathcal{U}) \subseteq G \cdot Z(\mathcal{U})$ in a more general setting. The obvious generalization of the torsion result would be again to prove that $\tilde{Z}(\mathcal{U}) \subseteq G \cdot Z(\mathcal{U})$. A weaker generalization of the torsion result would be to prove that $\tilde{Z}(\mathcal{U}) \subseteq G \cdot C_{\mathcal{U}}(T)$.

In the next section we prove (Theorem 2.3) that the second property holds whenever the torsion elements of $G$ form an Abelian subgroup $T$ and $\mathbb{Z}(G / T)$ has only trivial units. In the very particular case that $G / T$ is cyclic and every finite subgroup of $G$ is normal in $G$, we further obtain (Theorem 2.7) a complete description of $Z_{n}(\mathcal{U})$ for all $n \geq 1$ (in terms of $Z(\mathcal{U})$ ). In addition, we show that the stronger first property holds for various classes of groups.

The most substantial results in the paper are contained in section 3 and are concerned with the second centre $Z_{2}(\mathcal{U})$. Our main result there is Theorem 3.5, which characterizes $T$ whenever $Z_{2}(\mathcal{U})$ is not contained in $C_{\mathcal{U}}(T)$.

\section{Hypercentre}

We begin with two lemmas which will be required later. The proof of the first follows familiar lines but will be included for completeness.

\section{Lemma 2.1}

If $u$ is a hypercentral unit of finite order in $\mathbb{Z} G$ then $u$ is trivial.

\section{Proof}

Say $u \in Z_{n}(\mathcal{U})$ is of finite order. Since $u^{*} \in Z_{n}(\mathcal{U})$ is also of finite order, and the torsion elements of the nilpotent group $Z_{n}(\mathcal{U})$ form a subgroup, $u u^{*}$ must be of finite order. But $u u^{*}$ has nonzero identity coefficient, so $u u^{*}=1$ ([9], p.277). Hence $u \in \pm G$. 


\section{Lemma 2.2}

Assume the torsion elements of $G$ form a subgroup $T$. Then $\tilde{Z}(\mathcal{U}) \subseteq$ $N_{\mathcal{U}}(T)$.

\section{Proof}

We will prove by induction on $n$ that $Z_{n}(\mathcal{U}) \subseteq N_{\mathcal{U}}(T)$ for all $n \geq 1$. The case $n=1$ is obvious, so assume that the result holds for $n=k, k \geq 1$.

Let $u \in Z_{k+1}(\mathcal{U})$. If $t \in T$, then $u t u^{-1}=t \alpha$ for some $\alpha \in Z_{k}(\mathcal{U})$. By the induction hypothesis, $\alpha \in N_{\mathcal{U}}(T)$. Since $t^{m}=1$ for some $m, 1=u t^{m} u^{-1}=$ $\left(u t u^{-1}\right)^{m}=(t \alpha)^{m}=t_{1} \alpha^{m}$ for some $t_{1} \in T$. Thus $\alpha^{m} \in T$, and so $\alpha$ is a hypercentral unit of finite order. Lemma 2.1 says $\alpha \in \pm T$ and so $u \in N_{\mathcal{U}}(T)$ as desired.

The weaker property mentioned in the introduction now follows easily.

\section{Theorem 2.3}

Assume the torsion elements of $G$ form an Abelian subgroup $T$ and $\mathbb{Z}(G / T)$ has only trivial units. Then $\tilde{Z}(\mathcal{U}) \subseteq G \cdot C_{\mathcal{U}}(T)$.

\section{Proof}

Let $u \in \tilde{Z}(\mathcal{U})$. Lemma 2.2 tells us that $u \in N_{\mathcal{U}}(T)$, and thus $u g \in N_{\mathcal{U}}(T)$ for every $g \in G$. Since $\mathbb{Z}(G / T)$ has only trivial units, we know that $u g \in$ $1+(\mathbb{Z} G) \Delta T(*)$ for some $g \in G$. We will complete the proof by showing $u g \in C_{\mathcal{U}}(T)$.

Let $t \in T$ and $u g$ as above. Then $(u g) t(u g)^{-1}=t^{\prime}$, i.e. $(u g) t=t^{\prime}(u g)$ for some $t^{\prime} \in T$. This together with (*) gives that $t+\alpha t=t^{\prime}+t^{\prime} \alpha$ for some $\alpha \in(\mathbb{Z} G) \Delta T$. Focusing on elements of $T$, we get that $\left(t-t^{\prime}\right)(1+\beta)=0$ for some $\beta \in \Delta(T)$, i.e. $\left(1-t^{-1} t^{\prime}\right)(1+\beta)=0$. But this is impossible unless $t=t^{\prime}$ and we are done.

Our investigation of the stronger property $\tilde{Z}(\mathcal{U}) \subseteq G \cdot Z(\mathcal{U})$ proceeds via an intermediate step. We first show that $\tilde{Z}(\mathcal{U}) \subseteq N_{\mathcal{U}}(G)$ for a certain class of groups, and then note that the normalizer property $N_{\mathcal{U}}(G)=G \cdot Z(\mathcal{U})$ 
holds for these groups. Until recently it was an open problem as to whether this latter property holds in general. While a counterexample has recently been found [3], the property does hold for many groups and we use that here (see [9] for more detail on the normalizer problem).

\section{Lemma 2.4}

Let $G$ be a group with the property that whenever $x, y \in G$, there exists an integer $n$ (depending on $x$ and $y$ ) such that $x^{n}$ and $y$ commute. Then $\tilde{Z}(\mathcal{U}) \subseteq N_{\mathcal{U}}(G)$

\section{Proof.}

Using an argument similar to that seen in the proof of Lemma 2.2, we will prove by induction that $Z_{n}(\mathcal{U}) \subseteq N_{\mathcal{U}}(G)$ for all $n \geq 1$. If $n=1$, the result is obvious. So assume the result holds for $n=k, k \geq 1$, and let $u \in Z_{k+1}(\mathcal{U})$.

If $g \in G$, then $u g u^{-1}=g \alpha$ for some $\alpha \in Z_{k}(\mathcal{U})$. By the induction hypothesis, $\alpha \in N_{\mathcal{U}}(G)$. Since some power $g^{m}$ of $g$ commutes with $u$, we have

$$
g^{m}=u g^{m} u^{-1}=(g \alpha)^{m}=g_{1} \alpha^{m}
$$

for some $g_{1} \in G$.

So $\alpha^{m} \in G$, and thus $\alpha^{m}\left(\alpha^{m}\right)^{*}=1$. But $\alpha \in N_{\mathcal{U}}(G)$ implies $\alpha^{*} \alpha$ is central ([9], p.31), and so $\alpha^{*} \alpha=\alpha \alpha^{*}$. Thus we have $\left(\alpha \alpha^{*}\right)^{m}=1$ and so $\alpha \alpha^{*}$ is a central unit of finite order. Hence $\alpha \alpha^{*} \in G$, so $\alpha \in G$ and $u \in N_{\mathcal{U}}(G)$ as desired.

FC groups satisfy the conditions of Lemma 2.4. In the next result, parts (i) and (ii) could be stated more generally but we have opted for conciseness.

\section{Proposition 2.5}

The inequality $\tilde{Z}(\mathcal{U}) \subseteq G \cdot Z(\mathcal{U})$ holds in each of the following cases:

(i) $G$ is $\mathrm{FC}$ and has no 2-torsion.

(ii) $G$ is FC and is locally nilpotent. 
(iii) The torsion elements of $G$ form an Abelian subgroup $T$ contained in the FC subgroup of $G$ and $G=<T, g>$.

(iv) $G=T \rtimes X$ where $T$ is finite Abelian and $X$ is torsion free Abelian.

\section{Proof}

(i) and (ii) follow immediately from Lemma 2.4 and Theorem 2 of [4].

Assume we are in case (iii) and $u \in \tilde{Z}(\mathcal{U})$. Since the conditons of Lemma 2.4 are satisfied, $u \in N_{\mathcal{U}}(G)$. But now Theorem 1.4 of [4] says that $u=g^{i} u_{0}$ where $u_{0} \in \mathbb{Z} T$. Because $u_{0} g u_{0}^{-1} \in G$ and $u_{0} g u_{0}^{-1}-g \in[\mathbb{Z} G, \mathbb{Z} G], u_{0} g u_{0}^{-1}$ and $g$ must be conjugate in $G$ and hence in $T$. We have $u_{0} g u_{0}^{-1}=t_{0}^{-1} g t_{0}$ for some $t_{0} \in T$. Hence $t_{0} u_{0} \in Z(\mathcal{U})$ and we have our result.

Finally assume we are in case (iv) and $u \in \tilde{Z}(\mathcal{U})$. Again Lemma 2.4 says $u \in N_{\mathcal{U}}(G)$. Theorem 1.4 in [4] tells us that $u=g u_{0}$ where $u_{0} \in \mathbb{Z} T$ and $g \in G$. Let $H=C_{G}(T)$ and $K=H \cap X$. Observe that $K$ is a central subgroup of $G$ and that $\bar{G}=G / K \cong T \rtimes(X / K)$ is a finite metabelian group. Since $u_{0} \in C_{G}(T)$, we can conclude from Corollary 2.6 of [6] that conjugation in $\bar{G}$ by $\overline{u_{0}}$ is an inner automorphism. It follows that $\overline{u_{0} g_{0}^{-1}}$ is central in $\mathbb{Z} \bar{G}$ for some $g_{0} \in G$.

We claim that $u_{0} g_{0}^{-1}$ is a central unit in $\mathbb{Z} G$. Let $h \in G$. Since $u_{0} g_{0}^{-1} \in N_{\mathcal{U}}(G)$, we have seen that $\left[u_{0} g_{0}^{-1}, h\right] \in K$. However it is obvious that $\left[u_{0} g_{0}^{-1}, h\right] \in G^{\prime}$. Since $G^{\prime} \subseteq T$ and $T \cap K=1$, our result follows.

Note that while cases (iii) and (iv) are examples of the family of groups discussed in Theorem 2.3, cases (i) and (ii) give additional information about the weaker result $\tilde{Z}(\mathcal{U}) \subseteq G \cdot C_{\mathcal{U}}(T)$ as well. We conjecture that $\tilde{Z}(\mathcal{U}) \subseteq$ $G \cdot C_{\mathcal{U}}(T)$ holds whenever $G$ is an FC group.

The next lemma is needed to prove Theorem 2.7.

\section{Lemma 2.6}

(a) Assume $<t>\triangleleft G$ for any torsion element $t$ of $G$. Then if $u$ is a hypercentral unit in $\mathbb{Z} G, u t u^{-1}=t$ or $t^{-1}$ for any torsion element $t$ of $G$. Moreover, if $u t u^{-1}=t^{-1}$ then the order of $t$ is a power of 2 . 
(b) Assume $<t>\triangleleft G$ for any torsion element $t$ of $G$. Assume as well that the torsion elements of $G$ form an Abelian subgroup $T$ of $G$. Then if $u$ is a hypercentral unit in $\mathbb{Z} G$, either $u t u^{-1}=t$ for all $t \in T$ or $u t u^{-1}=t^{-1}$ for all $t \in T$. In the latter case, $T$ is a 2-group.

\section{Proof.}

(a) Note that the given condition implies that the torsion elements of $G$ form a subgroup $T$ of $G$.

Say $u \in Z_{n}(\mathcal{U})$ and $t \in T$. By Lemma 2.2 we know that $u t u^{-1} \in T$. Since $\overline{u t u^{-1}}=\overline{1}$ in $\mathbb{Z}(G /<t>)$, we conclude further that $u t u^{-1}=t^{i}$ for some integer $i, 1 \leq i \leq o(t)-1$. For convenience we set $l=\phi(o(t))$, where $\phi$ denotes the Euler phi function, in the rest of this proof.

Say $t^{i} \neq t, t^{-1}$. In that case

$$
b=\left(1+t+\cdots+t^{i-1}\right)^{l}+\frac{1-i^{l}}{o(t)} \hat{t}
$$

is a nontrivial Bass cyclic unit $([9]$, p.34) in $\mathbb{Z}<t>\subseteq \mathbb{Z} G$, and $b$ is of infinite order.

Note $b^{u}=\left(1+t^{i}+\cdots+t^{i(i-1)}\right)^{l}+\frac{1-i^{l}}{o(t)} \hat{t}$. In general,

$$
b^{u^{r}}=\left(1+t^{i^{r}}+\cdots+t^{i^{r}(i-1)}\right)^{l}+\frac{1-i^{l}}{o(t)} \hat{t}
$$

for any $r$.

It follows that $b b^{u} \cdots b^{u^{l-1}}$ is equal to $\left(1+t+\cdots+t^{i-1}\right)^{l}\left(1+t^{i}+\cdots+\right.$ $\left.t^{i(i-1)}\right)^{l} \cdots\left(1+t^{i^{i-1}}+\cdots+t^{i^{i-1}(i-1)}\right)^{l}+m \hat{t}$ for some integer $m$, and this product is equal to $\left(1+t+t^{2}+\cdots+t^{i^{l}-1}\right)^{l}+m \hat{t}=1+m_{1} \hat{t}$ for some integer $m_{1}\left(\right.$ since $\left.i^{l} \equiv 1(\bmod o(t))\right)$. Since $b$ has augmentation 1 , we conclude that $m_{1}=0$ and so

$$
b b^{u} \cdots b^{u^{l-1}}=1
$$

Now $u \in Z_{n}(\mathcal{U})$, so $b^{u^{r}} \equiv b\left(\bmod Z_{n-1}(\mathcal{U})\right)$ for all $r$, and we conclude that $b^{l} \in Z_{n-1}(\mathcal{U})$. 
Next observe that

$$
1=\left(b b^{u} \cdots b^{u^{l-1}}\right)^{l}=b^{l}\left(b^{l}\right)^{u} \cdots\left(b^{l}\right)^{u^{l-1}}
$$

since all $b^{u^{r}}$ are in $\mathbb{Z}<t>$ and thus they commute with one another. Since $b^{l} \in Z_{n-1}(\mathcal{U})$, we conclude as above that $b^{l^{2}} \in Z_{n-2}(\mathcal{U})$. Continuing we eventually get that $b^{s}=1$ for some $s \geq 1$, contradicting the fact that $b$ is of infinite order.

So $t^{i}=t$ or $t^{-1}$ as desired.

For the second part, observe that if $u \in Z_{n}(\mathcal{U})$ and $u t u^{-1}=t^{-1}$ then $t^{2} \in Z_{n-1}(\mathcal{U})$. Since $u t^{2} u^{-1}=t^{-2}, t^{4} \in Z_{n-2}(\mathcal{U})$. This process continues and gives the result.

(b) From (a) we know that if $u$ is a hypercentral unit and $t \in T$ then $u t u^{-1}=t$ or $t^{-1}$. Say we have $u t_{1} u^{-1}=t_{1}$ and $u t_{2} u^{-1}=t_{2}^{-1}$ for some $t_{1}, t_{2} \in T$ where $t_{1} \neq t_{1}^{-1}$ and $t_{2} \neq t_{2}^{-1}$. We know $u t_{1} t_{2} u^{-1}=t_{1} t_{2}^{-1}$. But we also must have $u t_{1} t_{2} u^{-1}=t_{1} t_{2}$ or $\left(t_{1} t_{2}\right)^{-1}$, and $t_{1} t_{2}^{-1}=t_{1} t_{2}$ and $t_{1} t_{2}^{-1}=t_{1}^{-1} t_{2}^{-1}$ both lead to a contradiction. Now the second part follows immediately from (a).

As mentioned in the introduction, when $G$ is torsion a complete description of $Z_{n}(\mathcal{U}) \cap G$ has been obtained for all $n$ (of course $Z_{n}(\mathcal{U})=Z_{2}(\mathcal{U})=$ $\tilde{Z}(\mathcal{U})$ for all $n \geq 2$ in this case). In general, if we are fortunate enough to have $\mathcal{U}(\mathbb{Z} G)=G \cdot Z(\mathcal{U})$ (as is the case when $\mathcal{U}$ is nilpotent, see Theorem $6.3 .23 \mathrm{in}[8])$ then it is easy to see that $Z_{n}(\mathcal{U})=Z_{n}(G) \cdot Z(\mathcal{U})$ for all $n>1$. We also have

\section{Theorem 2.7}

Assume that the torsion elements of $G$ form an Abelian subgroup $T$. In addition, assume every finite subgroup of $G$ is normal in $G$ and $G=<T, g>$.

(i) If $g t g^{-1}=t^{ \pm 1}$ for all $t \in T$, then $Z_{n}(\mathcal{U})=Z_{n}(G) \cdot Z(\mathcal{U})$ for all $n \geq 1$.

(ii) In all other cases, $Z_{n}(\mathcal{U})=\left(T \cap Z_{n}(G)\right) \cdot Z(\mathcal{U})$ for all $n \geq 1$. 


\section{Proof}

Note that the given conditions imply that $\mathcal{U}(\mathbb{Z} G)=G \cdot \mathcal{U}(\mathbb{Z} T)([8]$, Chapter VI).

Case (i) follows from above because under our assumption any unit $u$ in $\mathbb{Z} T$ can be written as $\alpha_{1} t$ where $\alpha_{1}$ is central ([9], p.10), and thus $\mathcal{U}(\mathbb{Z} G)=$ $G \cdot Z(\mathcal{U})$.

Now assume we are in case (ii). We proceed by induction on $n$. If $n=1$ we're done, so assume the result holds for $n=k, k \geq 1$.

If $t \in T \cap Z_{k+1}(G)$, then $t g t^{-1}=g t^{j} \quad$ (since $<t>\triangleleft G$ ) where $t^{j} \in$ $T \cap Z_{k}(G)$. The induction hypothesis says that $t^{j} \in Z_{k}(\mathcal{U})$ and, using $\mathcal{U}(\mathbb{Z} G)=G \cdot \mathcal{U}(\mathbb{Z} T)$, we conclude that $t \in Z_{k+1}(\mathcal{U})$, giving containment in one direction.

So now consider the other containment. Proposition 2.5 tells us that $Z_{k+1}(\mathcal{U}) \subseteq G \cdot Z(\mathcal{U})$, and the proof will be completed if we show that the first term in the product on the right can be chosen in $T \cap Z_{k+1}(\mathcal{U})$, since it is easy to see that $G \cap Z_{n}(\mathcal{U}) \subseteq Z_{n}(G)$ for all $n \geq 1$. To this end, assume a typical group element $t_{1} g^{k}, t_{1} \in T$, belongs to $G \cap Z_{k+1}(\mathcal{U})$. It follows from Lemma 2.6(b) that $\left(t_{1} g^{k}\right)^{-1} t\left(t_{1} g^{k}\right)=g^{-k} t g^{k}$ equals $t$ or $t^{-1}$ for all $t \in T$. If $g^{-k} t g^{k}=t$ were true for all $t$ then $g^{k}$ would be central and we would be done. So we can assume that $t_{1} g^{k} \in G \cap Z_{k+1}(\mathcal{U})$ and $g^{-k} t g^{k}=t^{-1}$ for all $t \in T$ (and also $T$ is a 2-group).

Since we are not in case (i), there must exist $t_{0} \in T$ such that $g t_{0} g^{-1}=t_{0}^{l}$ where $l$ is odd, $1<l<o\left(t_{0}\right)-1$. It follows that $o\left(t_{0}\right)=2^{m}$ for some $m \geq 3$.

Also $g t_{0} g^{-1}=t_{0}^{l}$ and $g^{k} t_{0} g^{-k}=t_{0}^{-1}$ imply that $k$ must be even (the order of $g$ as an automorphism of $<t_{0}>$ must divide $\phi\left(2^{m}\right)=2^{m-1}$ and be

$\geq 4$, while $2 k$ is divisible by this order). Now $g^{k} t_{0} g^{-k}=t_{0}^{l^{k}}$, so $2^{m}$ divides $1+l^{k}$. But $l^{k} \equiv 1(\bmod 4)$, contradicting the fact that $o\left(t_{0}\right)$ is divisible by 4. We conclude that this situation cannot occur, and the proof is complete.

\section{Second Centre}

All results so far have concerned $Z_{n}(\mathcal{U})$ where $n$ is any natural number. As mentioned earlier, when $G$ is torsion this is equivalent to proving results 
about $Z_{2}(\mathcal{U})$, but this is not true in general. Some of the observations made earlier can be sharpened in the particular case $n=2$. Our main result is Theorem 3.5 which characterizes $T$ whenever $Z_{2}(\mathcal{U})$ is not contained in $C_{\mathcal{U}}(T)$.

The first result demonstrates a sharpening of Lemma 2.6.

\section{Lemma 3.1}

(a) If $t \in G$ is of finite order and $u \in Z_{2}(\mathcal{U})$, then $u t u^{-1}=t$ or $t^{-1}$. Moreover, if $u t u^{-1}=t^{-1}$ then the order of $t$ divides 4 .

(b) Assume the torsion elements of $G$ form an Abelian subgroup $T$ of $G$. Then if $u \in Z_{2}(\mathcal{U})$, either $u t u^{-1}=t$ for all $t \in G$ or $u t u^{-1}=t^{-1}$ for all $t \in T$. In the latter case, the exponent of $T$ divides 4 .

\section{Proof.}

(a) Let $u, t$ be as stated, and assume $t^{n}=1$. Note that $u t u^{-1}=t z$ where $z \in Z(\mathcal{U})$, and $t^{n}=1$ implies $z^{n}=1$ also, so $z \in T$ and $u^{n}$ commutes with $t$.

Consider the unipotent unit $v=1+(1-t) u \hat{t}$. We know that $u^{n}$ commutes with $v$. On the other hand $u v=v u c$ for some $c \in Z(\mathcal{U})$, so $c^{n}=1$. It follows that $u$ commutes with $v^{n}$. But $v^{n}=1+n(1-t) u \hat{t}$, and we conclude that $u$ commutes with $v$.

So $u(1-t) u \hat{t}=(1-t) u \hat{t} u$, or $(1-t) u \hat{t} u^{-1}=\left(1-u^{-1} t u\right) \hat{t}$.

Earlier in the proof we observed that $u t u^{-1}$ and $u^{-1} t u$ are both in $T$. It follows that $u t u^{-1} \in<t>$.

Now exactly the same argument as in the proof of Lemma 2.6(a) can be used. If $u t u^{-1}=t^{i} \neq t, t^{-1}$, we can construct a nontrivial Bass cyclic unit

$$
b=\left(1+t \cdots+t^{i-1)^{\phi(o(t))}}+\frac{1-i^{\phi(o(t))}}{o(t)} \hat{t} .\right.
$$

We have $b b^{u} \cdots b^{u^{\phi(o(t))-1}}=1$, and this leads to the contradiction that $b$ is of finite order. 
So $u t u^{-1}=t$ or $t^{-1}$ as desired. The last statement follows from $u t u^{-1}=t\left(t^{-2}\right)$, which forces $t^{2}$ to be central.

(b) This follows in exactly the same way as in the proof of Lemma 2.6(b).

We require the following observation concerning unipotent units.

\section{Lemma 3.2}

Assume $t \in G$ is of finite order and $u \in Z_{2}(\mathcal{U})$. Then for any $\alpha \in \mathbb{Z} G, u$ commutes with the unipotent unit $v=1+(1-t) \alpha \hat{t}$.

\section{Proof.}

We know $u v=v u c$ for some $c \in Z(\mathcal{U})$. So $u v^{2}=v^{2} u c^{2}$.

But $v^{2}=1+2(1-t) \alpha \hat{t}=2 v-1$. Substituting we get $u(2 v-1) u^{-1}=$ $(2 v-1) c^{2}$, or $2 v c-1=2 v c^{2}-c^{2}$. This means that

$$
2(1-v)\left(c^{2}-c\right)=2\left(c^{2}-c\right)+\left(1-c^{2}\right)=(c-1)^{2} .
$$

Note that $(1-v)^{2}=0$, so we get $(c-1)^{4}=0$. Since $\mathbb{Z} G$ contains no nonzero central nilpotent elements, this gives $c=1$ and we're done.

The following technical lemma will be needed in what follows.

\section{Lemma 3.3}

Assume that the set of torsion elements of $G$ is a subgroup $T$ of $G$ and $<t_{1}>\pitchfork T$ for some $t_{1} \in T$. Then $[t, u] \in<t_{1}>$ for all $u \in Z_{2}(\mathcal{U})$ and all $t \in T$. In addition, either $[t, u]=1$ or $[t, u]$ is the unique (central) element of order 2 in $\left\langle t_{1}>\right.$ and, in the latter case, $t^{2}=[t, u]$.

\section{Proof.}

Choose $u \in Z_{2}(\mathcal{U})$ and $t \in T$.

First assume that $t t_{1} t^{-1} \notin<t_{1}>$. In that case, $v=1+\left(1-t_{1}\right) t^{-1} \hat{t_{1}}$ is a nontrivial bicyclic unit and Lemma 3.2 tells us that $u v u^{-1}=v$. So $1+\left(1-u t_{1} u^{-1}\right)\left(u t^{-1} u^{-1}\right) \hat{t_{1}}=1+\left(1-t_{1}\right) t^{-1} \hat{t_{1}}$. Again using Lemma 3.1, we conclude that either $[t, u]=1$ or $u t u^{-1}=t^{-1}$ and, in the latter case, $t=t^{-1} t_{1}^{i}$ for some $i$ and $[t, u]=t^{2}=t_{1}^{i}$ is central of order 2 . 
Next assume that $t t_{1} t^{-1} \in<t_{1}>$. We know there exists $t_{2} \in T$ such that $t_{2} t_{1} t_{2}^{-1} \notin<t_{1}>$ and the above paragraph tells us that $\left[t_{2}, u\right] \in<t_{1}>$. Similarly $\left(t_{2} t\right) t_{1}\left(t_{2} t\right)^{-1} \notin<t_{1}>$ implies $\left[t_{2} t, u\right] \in<t_{1}>$. Since $\left[t_{2}, u\right]$ and $\left[t_{2} t, u\right]$ are both central, $[t, u] \in<t_{1}>$ also and the other results follow easily.

One of the questions completely settled for torsion groups in [7] is that of determining when $Z_{2}(\mathcal{U}) \neq Z(\mathcal{U})$. Information on this for more general groups can be deduced from the earlier results as well as the following example, in which a family of groups with $Z_{2}(\mathcal{U}) \neq Z(\mathcal{U})$ are constructed.

\section{Example 3.4}

Let $A$ be an Abelian group containing an element $h$ of order 4 . Let $G=<A, x \mid x a x^{-1}=a^{-1}$ for all $a \in A, x^{2}=h^{2}>$, i.e. $G$ is like a $Q^{*}$-group but may contain elements of infinite order. Then the argument outlined in the first paragraph of the proof of Theorem 2 in [7] shows that $h \in Z_{2}(\mathcal{U})$ but $h \notin Z(\mathcal{U})$.

We will close with our main result, which deals with the weaker question of when $Z_{2}(\mathcal{U}) \nsubseteq C_{\mathcal{U}}(T)$.

\section{Theorem 3.5}

Assume that the set of torsion elements of $G$ is a subgroup $T$ of $G$. If $Z_{2}(\mathcal{U}) \nsubseteq C_{\mathcal{U}}(T)$, then $T$ is either an Abelian group of exponent 4 or a $Q^{*}$-group.

\section{Proof.}

If $T$ is Abelian, we know from Lemma 3.1(b) that it must be of exponent 4 .

Assume $T$ is Dedekind group, i.e. $T \cong K_{8} \times E_{2} \times E_{2}^{1}$ where $E_{2}$ is an elementary Abelian 2-group and $E_{2}^{1}$ is a group of odd order. Let $u \in$ $Z_{2}(\mathcal{U}) \backslash C_{\mathcal{U}}(T)$. Then Lemma 3.1(a) says $u t u^{-1}=t$ for all $t \in E_{2} \times E_{2}^{1}$, so there exists $x \in K_{8}$ of order 4 such that $u x u^{-1}=x^{3}$. Say it is possible to choose $y \neq 1$ in $E_{2}^{1}$ of prime order $p$. Since $x y$ is of order $4 p, u x y u^{-1}=$ $x y$. But $u x y u^{-1}=x^{3} y$. This contradiction tells us that $T \cong K_{8} \times E_{2}$, a Hamiltonian 2-group, and this is a $Q^{*}$-group. 
So assume now that there exists $t_{1} \in T$ with $<t_{1}>\pitchfork T$. Again let $u \in Z_{2}(\mathcal{U}) \backslash C_{\mathcal{U}}(T)$. Let $H=\{t \in T \mid[t, u]=1\}$. We know $H \neq T$ and, using Proposition 4.1(a), it is easy to see that $H \triangleleft T$. Moreover, if $x, y \in T \backslash H$ then $[x, u]=[y, u]$ is central of order 2 by Lemma 3.3 , so $[x y, u]=1$ and $x y \in H$. Thus $|T / H|=2$.

If $a \in T \backslash H$ and $x \in H$, then $a^{2}=(a x)^{2}$ by Lemma 3.3 so $a x a^{-1}=x^{-1}$. If in addition $y \in H$ then $a y a^{-1}=y^{-1}$ and $a x y a^{-1}=(x y)^{-1}=y^{-1} x^{-1}$. So $x^{-1} y^{-1}=y^{-1} x^{-1}$ and $H$ is Abelian.

Finally, observing that $T / H^{2}$ is Abelian we conclude that if $[t, u] \neq 1$ then $[t, u] \in H^{2}$. This completes the proof that $T$ is a $Q^{*}$-group.

It is easy to see that both possibilities can occur. If $G$ is a $Q^{*}$-group, then $Z_{2}(\mathcal{U}) \neq Z(\mathcal{U})\left([7]\right.$ or Example 3.4) and also $G=T$, so $Z_{2}(\mathcal{U}) \nsubseteq C_{\mathcal{U}}(T)$. Also, if $G=<t, g \mid t^{4}=1, g t g^{-1}=t^{3}>$ then Theorem 2.7 says that $t \in$ $Z_{2}(\mathcal{U})$.

\section{ACKNOWLEDGEMENTS}

The authors wish to express their thanks to the referee for his suggestions in preparing the revised version of the manuscript.

\section{REFERENCES}

1. Arora, S. R.; Hales, A.W.; Passi, I.B.S. Jordan decomposition and hypercentral units in integral group rings. Comm. Alg. 1993, 21, 25-35.

2. Arora, S. R.; Passi, I.B.S. Central height of the unit group of an integral group ring. Comm. Alg. 1993, 21, 3673-3683.

3. Hertweck, M. A counterexample to the isomorphism problem for integral group rings. Ann. of Math. 2001, 154, 115-138.

4. Jespers, E.; Juriaans, S.O.; de Miranda, J.M.; Rogerio, J.R. On the normalizer problem. J. Algebra 2002, 247, 24-36.

5. Li, Y. The hypercentre and $n$-centre of the unit group of an integral group ring. Canad. J. Math. 1998, 50, 401-411. 
6. Li, Y. The normalizer of a metabelian group in its integral group ring. J. Algebra (to appear).

7. Li, Y.; Parmenter, M.M. Hypercentral units in integral group rings. Proc. Amer. Math. Soc. 2001, 129, 2235-2238.

8. Sehgal, S.K. Topics in Group Rings; Marcel Dekker: New York, 1978.

9. Sehgal, S.K. Units in Integral Group Rings; Longman: Essex, 1993. 